\section{Automated Delineation Of Wildfire Areas Using Sentinel-2 Satellite Imagery}

Gl_Forum 2018, Issue.1

Page: 251 - 262

Full Paper

Corresponding Author:

weiratherm@yahoo.de

DOI: 10.1553/giscience2018_01_s251

\author{
Mira Weirather', Gunter Zeug' and Thomas Schneider² \\ 'Terranea, Germany \\ 2Technical University of Munich, Germany
}

\begin{abstract}
Climate change will bring many changes to the world. For example, the frequency and severity of natural hazards and related disasters are expected to increase globally. Wildfires already affect thousands of people every year and cause billions of Euros' worth of damage. It is therefore paramount to develop measures that help deal with the consequences of wildfires. Forests being the largest terrestrial ecosystem in the European Union and providing many ecosystem services, their loss due to wildfires is of serious concern. In this study, an algorithm to extract the burned area of wildfire events is presented. It was developed on the basis of three fire events in 2017. The procedure is fully automated, from downloading suitable data to determining the burned area by applying the differenced Normalized Burn Ratio (dNBR) on open Sentinel-2 satellite imagery from the European Copernicus programme. First results show good performance and encourage its further development and application. It is planned that the output of our mapping will feed into and be used in calibrating wildfire simulations during longer fire events.
\end{abstract}

\title{
Keywords:
}

wildfires, Sentinel-2, Copernicus, cloud computing

\section{Introduction}

According to MunichRe (2018), the majority of disasters during the last decade, including floods, storms, heatwaves and droughts, were caused by natural hazards linked to climate and weather. 2017 was the year of the highest insured natural disaster losses ever, amounting to US\$135bn. Overall losses, i.e. including uninsured losses, amounted to US\$ 330bn, the second-highest figure ever recorded for natural disasters (MunichRe, 2018). Extreme weather in conjunction with the increasing numbers and sizes of built-up areas in disaster-prone regions is one reason for such high figures. It is expected that the likelihood of extreme weather events will increase due to global climate change (IPCC, 2013). As a consequence, natural disasters such as wildfires will be a greater threat to the environment and to society. For Europe, it is projected that areas which are currently safe from fires will become more prone to them, and that fire seasons will be longer (Moreno, 2014). 
These new challenges need to be taken into account by authorities and call for new measures for dealing with wildfires. The aim of the work presented in this paper is to develop a fully automated algorithm capable of extracting the exact burned area during and after a wildfire event. Due to the large amounts of data provided by today's Earth observation satellites, automated cloud-based processing approaches are required that can fully exploit the value of the available data. This research is based solely on open source tools for processing, and only on open data. The algorithm could be used by insurance companies to support their loss adjustments. We are also planning to use the output of the approach to feed into and calibrate wildfire simulations during longer fire events.

Our work is part of I-REACT, a European research project funded through the European Union's Horizon 2020 research and innovation programme. At the time of writing this paper, the project is still ongoing. The objective at the core of the I-REACT project is to combine data from multiple sources to enable a quicker response to catastrophes such as wildfires. Data coming from new technological developments created by I-REACT, such as a mobile app for real-time crowdsourced information, drones, wearables and augmented reality glasses, is combined with data from weather forecasts, European monitoring systems, earth observation and historical information. Thus, citizens are involved, and policy makers can be supported, leading to the possibility of early warning and response (http://www.ireact.eu/).

\section{Scientific Background}

Forests are the largest terrestrial ecosystem in the EU, amounting to more than $40 \%$ of the total land cover (Barredo et al., 2015). They provide many ecosystem services, such as nutrient cycling and air quality regulation (Barredo, 2014); their great contribution to the environment and to society is lost when they are destroyed by fire.

To prevent the breakout of a fire, it is necessary to deal with the causes of wildfires. According to the 'Harmonized classification scheme of fire causes in the EU' developed by the JRC, six categories of causes can be distinguished, subdivided into 29 classes (Camia et al., 2013). For example, from 1985 to 2010 the most frequent fire cause (54\% of cases) in Spain was arson, followed by unknown reasons (22\%) and negligent use of fire (14\%); $4 \%$ of fires had natural causes (Camia et al., 2013). Such a classification can enable authorities to take preventative measures tailored to particular fire causes, such as introducing more severe penalties for the negligent use of cigarettes or fireworks, or improving the condition of electrical power lines or railways. While causes such as accidents, negligence or arson can be addressed to lower the incidence of fires, natural causes of fire are more difficult to counter. In fact, given that our climate is changing, the percentage of naturally caused fires is likely to increase. Meteorological conditions will change towards those favouring fire ignition as the average temperature as well as the frequency of heat waves increase (Moreno, 2014).

Remote sensing is the standard tool used to delineate affected burned areas. As part of the MODIS Active Fire and Burned Area Products, burned areas are mapped on a global scale, investigating spectral, temporal and structural changes (Roy et al., 2005). The application of various spectral indices such as the Normalized Difference Vegetation Index (NDVI), 
Burned Area Index (BAI), and Normalized Burn Ratio (NBR) have been widely used to monitor fire-induced vegetation changes (Chen et al., 2011; Veraverbeke et al., 2011). Sentinel-2 imagery from the European Copernicus programme now provides the ideal tool to map and monitor wildfires and their evolution globally, especially because of its higher spatial resolution, of $10 \mathrm{~m}$ to $60 \mathrm{~m}$. With a revisit rate of five days and spectral resolution in 13 bands, the data provided by the satellites allows the reliable processing of the abovementioned indices.

\section{Methodology}

\section{Burn Index}

In this study, the differenced Normalized Burn Ratio (dNBR) is used for the extraction of burned areas. It is calculated from the Normalized Burn Ratio (NBR) of an image before and after the fire. The NBR was developed for Landsat TM/ETM+ making use of the nearinfrared (NIR) and short-wave-infrared (SWIR) bands for its calculation, as shown in the following formula:

$$
N B R=\frac{N I R-S W I R}{N I R+S W I R}
$$

Healthy vegetation shows high reflectance in the near-infrared range, and low reflectance in the shortwave infrared range. Conversely, burned areas show low reflectance in the nearinfrared but high reflectance in the shortwave infrared bands. Therefore, high NBR values indicate unburned vegetation, while low values indicate burned areas (Key \& Benson, 2006). In the case of Sentinel-2, the bands closest to the original TM/ETM+ bands were selected. These were band 12 for SWIR, and band 8 or $8 \mathrm{~A}$ for NIR. Since bands 12 and $8 \mathrm{~A}$ both have a resolution of $20 \mathrm{~m}$, band $8 \mathrm{~A}$ was chosen over band 8 , which has a $10 \mathrm{~m}$ resolution. NBR was chosen over BAI, which is based on the reflectance in the red and near-infrared channels (Chuvieco et al., 2002), because NBR gave better results in several tests conducted prior to implementation. The study of Huang et al. (2016) supports this approach.

\section{Calculation of thresholds}

NBR values derived from satellite remote sensing vary due to different image characteristics and image-acquisition conditions; field visits would usually help in choosing suitable thresholds to distinguish between burned and unburned areas. However, the aim is to develop an automated approach applicable to the whole EU that does not require field assessments. Consequently, universal thresholds for classifying burned areas would be ideal. It was therefore decided to classify thresholds for burned and unburned areas for different biogeographical regions with similar characteristics of habitats and species, irrespective of national borders (EEA, 2002). Since the area of particular interest is in central Europe, six of the largest biogeographical regions were chosen for the calculations, i.e. Alpine, Atlantic, Boreal, Continental, Mediterranean and Pannonian. For each region, dNBR values were calculated for several fire events from 2017 and matched with burned areas taken from the 
database of the European Forest Fire Information System (EFFIS). Averages of the resulting thresholds were calculated and applied in the algorithm. To further approximate optimal thresholds, we are in the process of reanalysing more historical fire events from EFFIS.

\section{Study Area}

To test the algorithm, three fire events in the EU were chosen as test areas. The study areas were selected from registered events of the Copernicus Emergency Management Service (EMS), which allowed us to use the EMS maps as reference for the accuracy assessment. The 2017 fire season was chosen. In 2017, the EMS mapped 21 fire events in the EU, occurring in six countries: Spain (11 events), France (3), Greece (2), Italy (2), Portugal (2) and Hungary (1). According to the map of biogeographical regions of Europe (EEA, 2018), 18 of these events took place in the Mediterranean region, one in the Pannonian region (Hungary), and two in the Alpine region (Italy, Spain). To ensure the broadest diversity possible of vegetation types with the given data, one event in the Mediterranean region (Greece, EMSR242), one in the Alpine region (Spain, EMSR259) and one in the Pannonian region (Hungary, EMSR230) were selected (Figure 1).

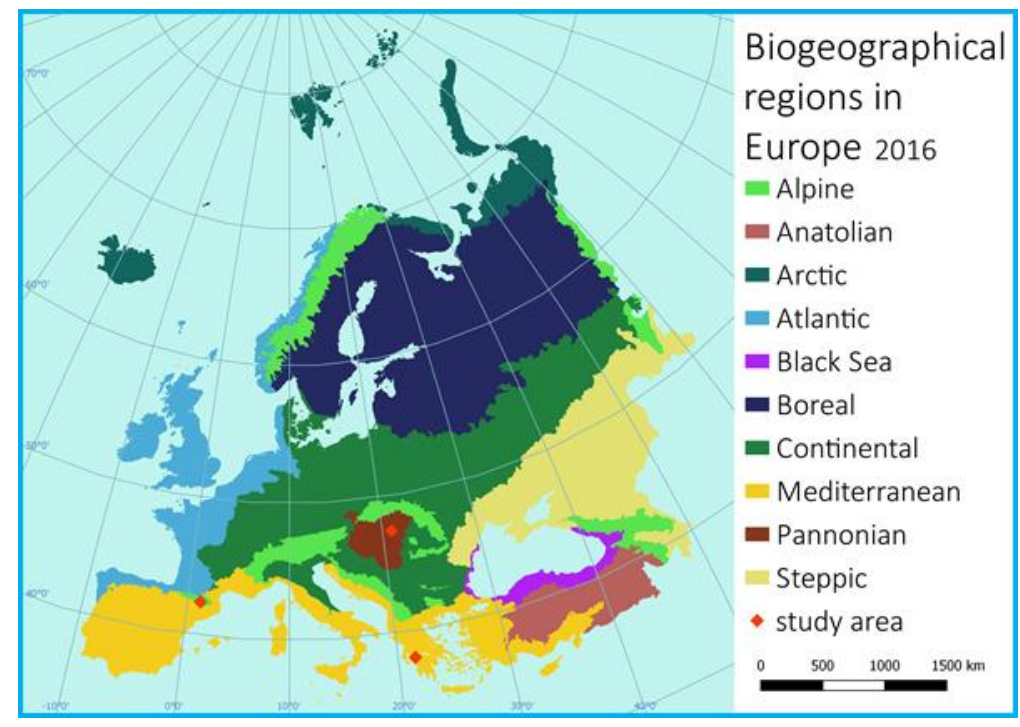

Figure 1: Biogeographical regions in Europe 2016 based on (EEA, 2018)

\section{Processing in the Cloud}

\section{The Trigger}

In the planned workflow, maps of burned areas are generated only when they are requested directly by an authorized user executing a 'Map Request' in a web portal. This request triggers the activation of the algorithm in the backend. The trigger contains the coordinates of the area of interest (AOI) and the date when the fire started. A service is set up to 
continuously listen for new map requests on the server side. When processing is finished, a cron job re-starts the listener.

\section{Automated Workflow}

The first step in the automated process is to download suitable imagery from Amazon Web Services (AWS). AWS was chosen over other Copernicus image hubs because it is also the cloud environment used for the service implementation and it saves resources for up- and downloading imagery. The algorithm uses Sentinel-2 imagery as input. Both a pre-event image and a post-event image are downloaded. The trigger contains AOI coordinates as well as the time period for which imagery is required. For the selection of a pre-event image, data is searched in a 10-day period ending with the day before the outbreak of the fire. Since the process is only triggered on demand, the time period to search for suitable post-event images starts on the day of the trigger. If the fire started more than 10 days before the demand time, the search period is extended backwards by periods of 10 days at a time. The cloud mask of the least clouded scene is intersected with the AOI, and the process checks whether the location of the fire is cloudy. If this is the case, the next results are checked. If still no suitable scene can be found, the time period is extended until a scene with no clouds in the AOI is found. For the pre-event image, this means shifting the start time backwards. For the post-event image, the search is repeated a few days later, when new images become available.

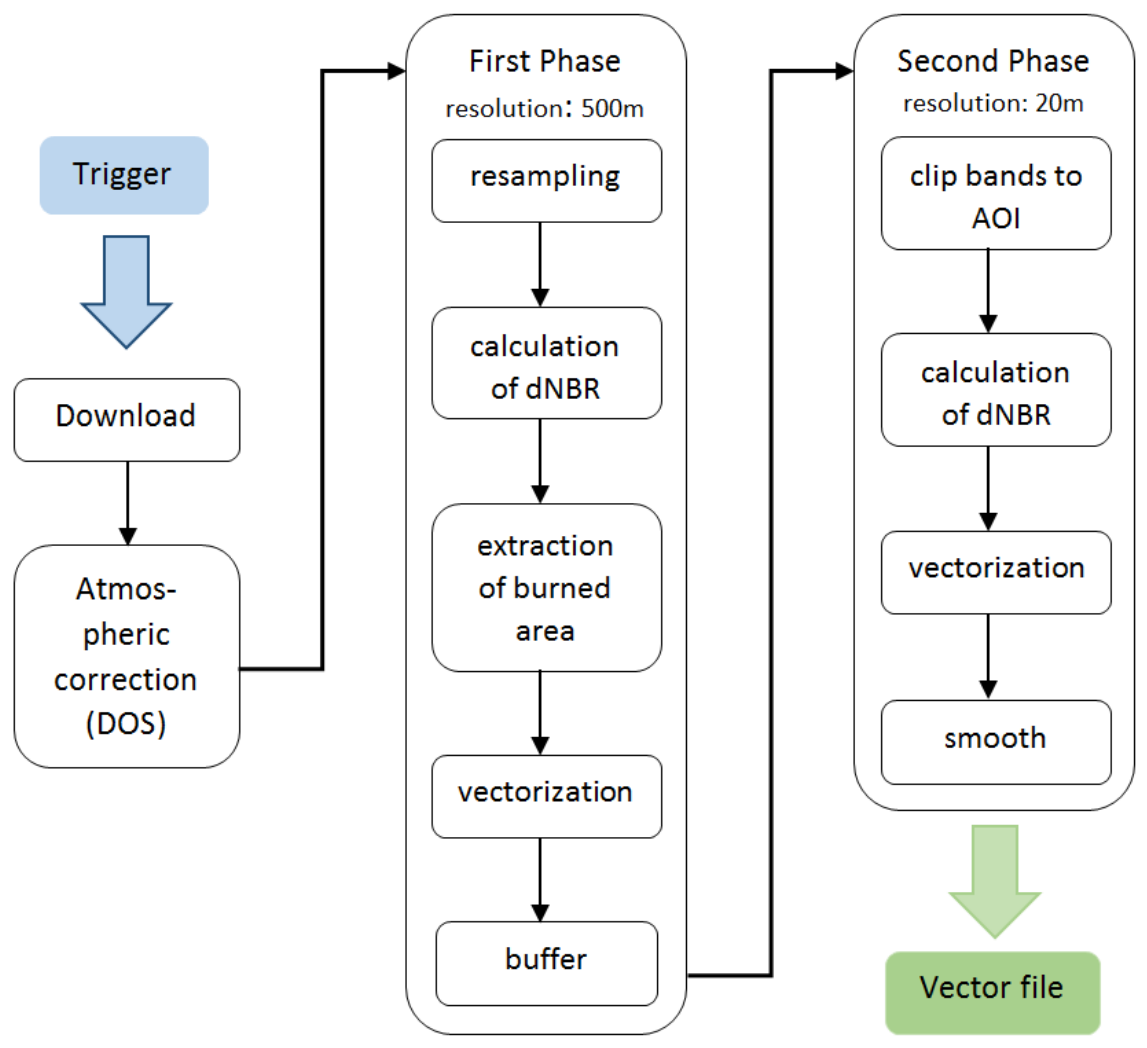

Figure 2: Workflow of the fully automated algorithm 
Figure 2 shows the overall processing workflow. After the trigger, suitable pre- and postevent images are downloaded and committed to the next module, in which the preprocessing takes place. There, the necessary Sentinel-2 bands (B8A, B12) containing TOA reflectances are calibrated to surface reflection by a Dark Object Subtraction (DOS) based atmospheric correction (Chavez, 1988). DOS was chosen because of its robustness and ease of implementation. More sophisticated approaches for atmospheric correction such as Sen2Cor were not tested since they significantly influence only the separability of the visible wavelength bands (Huang et al., 2016).

The next step is to extract the burned area using a two-phase algorithm. In the first phase, the algorithm is executed, with the bands being resampled at a lower resolution to obtain an initial mask of the AOI affected. Therefore, the $20 \mathrm{~m}$ bands are resampled at $500 \mathrm{~m}$ resolution. Based on this, preNBR and postNBR are calculated; postNBR is then subtracted from preNBR to produce the dNBR. Finally, a threshold is applied to obtain the burned area only (Figure 3). In the current approach, adequate thresholds were chosen after assessing three fire events, one in each of the three different biogeographical regions. However, it is planned to further identify and narrow down suitable thresholds following the analysis of other wildfire events. Pixels for burned areas show the highest values since they changed the most within the time period from pre- to post-image. Nevertheless, a relatively low threshold is used to create a connected area of the pixels for the burned areas. Next, physically discrete pixels are grouped into unique categories. After extracting the relevant pixels, the raster is vectorized. In order to also cover smaller burned areas at the edges of the fire event, where the fire may have rekindled, a polygon buffer is applied.

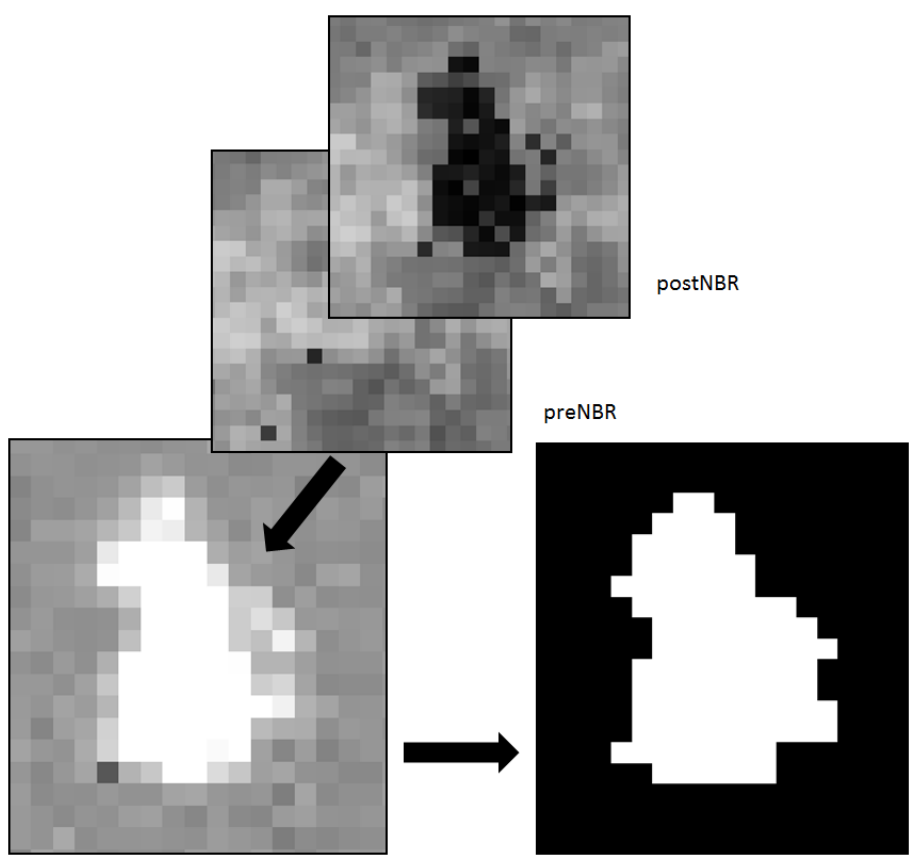

Figure 3: PostNBR and preNBR are calculated and postNBR subtracted from preNBR, resulting in the dNBR. Applying the threshold value leads to the burned area. 
In the second processing phase, the burned area is extracted using the original $20 \mathrm{~m}$ bands but clipped to the extent of the buffered polygon obtained from phase one. Again, preNBR, postNBR and dNBR are calculated. In this phase, the threshold used to identify the pixels for the burned area is higher, because a more detailed and accurate result for the area is needed. The resulting raster layer can be vectorized directly, allowing the algorithm to identify smaller burned areas in addition to the main event. In order to obtain a more appealing outline of the resulting vector, smoothing is applied. The output generated is a vector, which is returned to the central computing backend of the project. Cloud computing, namely the Amazon Elastic Compute Cloud (Amazon EC2), is used for the processing of the complete algorithm, allowing it to start independently and without human interaction when activated by the trigger. This fulfils the need for a fully automated process. The algorithm is implemented using the programming language Python. Furthermore, it utilizes open source tools from QGIS, GDAL, SAGA GIS and GRASS GIS.

\section{$4 \quad$ Results}

A visual comparison of initial results obtained with the algorithms and overlaid with the Copernicus EMS reference maps shows that the approach delivers accurate results. Figures 4 -6 show the post-event imagery of the three test areas compared to the EMS results. In the false-colour images, the burned land is visible as blackened areas. Error matrixes of the results were calculated for each AOI using the results of the EMS as reference maps. A total of 1,000 random points were used, and overall accuracy $(\mathrm{OA})$, producer's accuracy (PA), user's accuracy (UA) and Cohen's K were calculated (Congalton et al., 2009). 


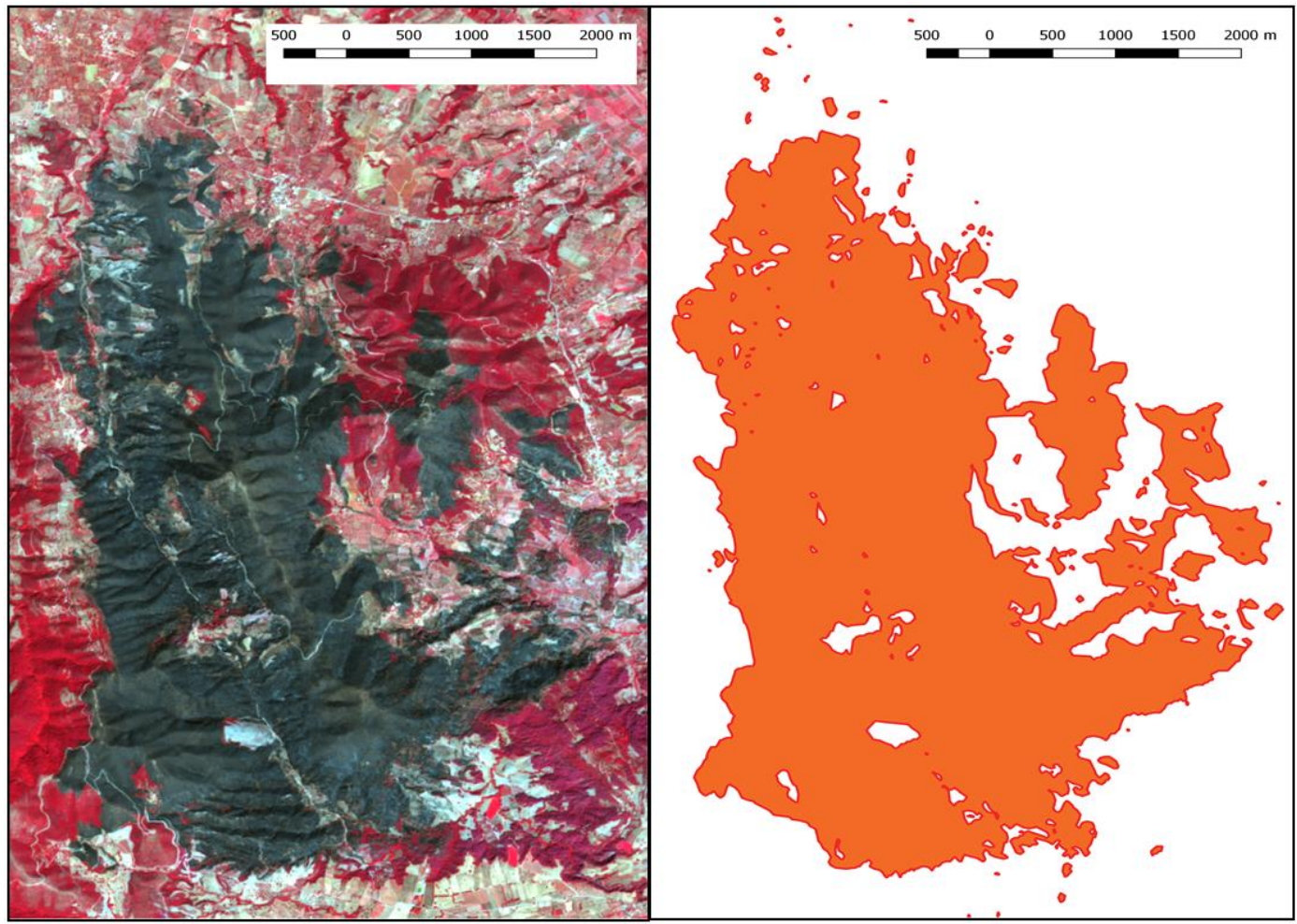

Figure 4: Post-fire false-colour image (bands 8, 4,3) and Burned Area Map for EMSR242. The blackened area represents charred vegetation.

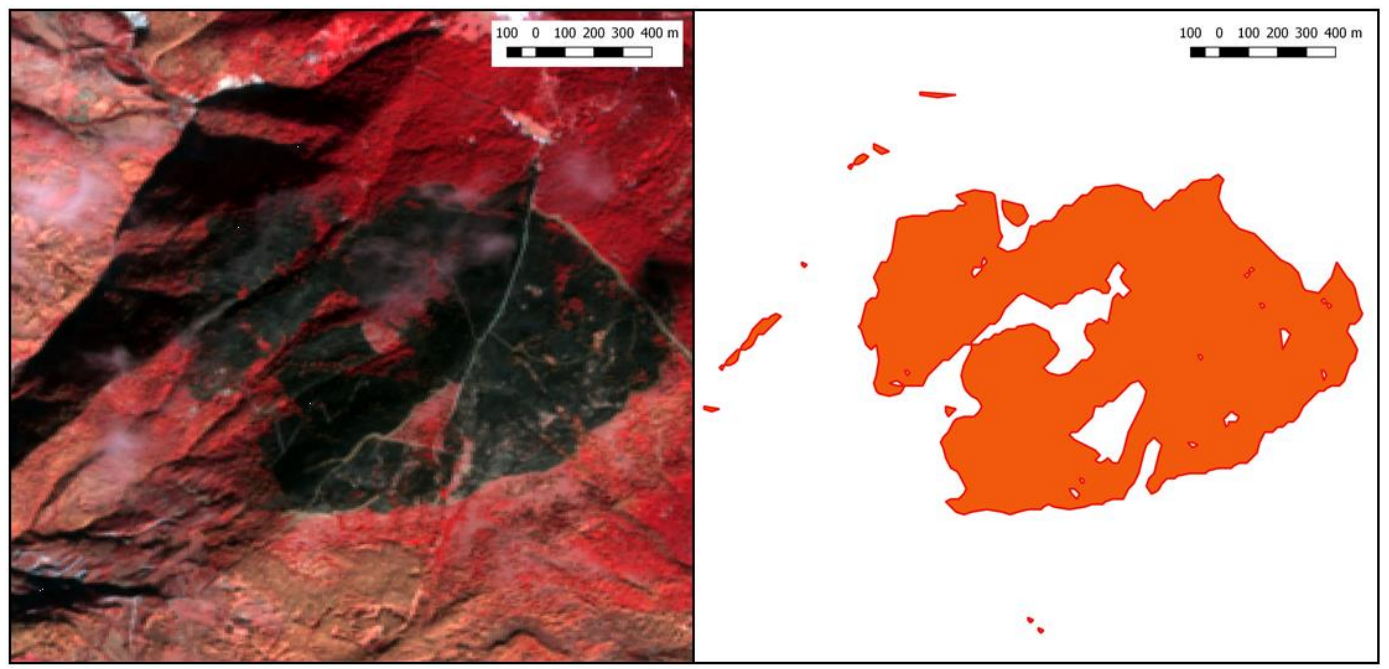

Figure 5: Post-fire false-colour image (bands 8, 4, 3) and Burned Area Map for EMSR259. The blackened area represents charred vegetation. 


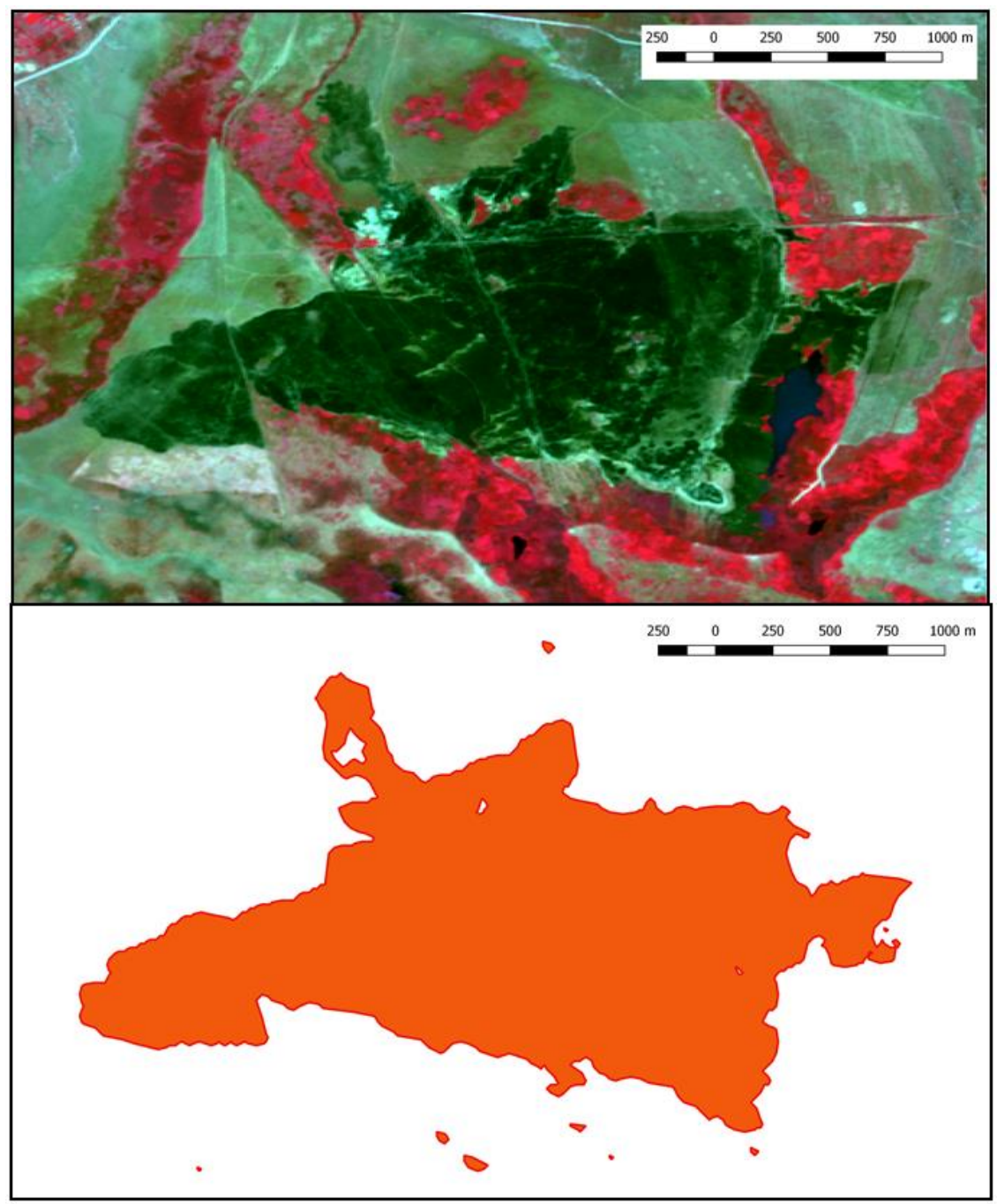

Figure 6: Post-fire false-colour image $(\underline{b a n d s} 8,443)$ and Burned Area Map for EMSR230. The blackened area represents charred vegetation.

The results of the error matrix are shown in Table 1. Overall accuracy of the burned area maps reached values of 0.97 for EMRS242 and EMSR230, and 0.96 for EMSR259, and Kappa coefficients of 0.93 and 0.90 . Through a visual check, it could be seen that the EMS reference map for EMSR242 includes burned areas from an earlier fire event. With the approach presented here, we were able to separate these events correctly. The good results we obtained confirm that calculating region-specific thresholds is a sensible approach. The NBR is able to detect black pixels for burned or charred vegetation (Figure 3) very well. Due to the wide variety of vegetation and landscape patterns in a burned area (char, burned leaves or lightly burned leaves), specific spatial patterns for burned patches cannot be recognized (Smith et al., 2002). With the algorithm, even island polygons can be detected as burned 
areas (outside the main event) or unburned ones (inside the main event). The classification of lightly or partly burned areas is more challenging and influenced by the chosen threshold values. The higher the value, the fewer grey pixels are added to the burned area, resulting in a smaller area, and vice versa. One way of handling this would be to first choose a low threshold value to obtain a larger burned area and to further classify this area according to its burn severity, e.g. by creating classes for unburned, lightly burned, moderately burned and severely damaged areas. To include context in the classifier is another possibility to better handle the lightly or partly burned areas. The two-phase procedure proves to be a good approach also for detecting smaller burned areas around the main affected area. Furthermore, the approach accelerates the processing, as it only searches for pixels for burned vegetation in the AOI generated in the first phase rather than in the whole scene.

Table 1: Accuracy measurements of burned area maps^

PA: producer's accuracy

UA: user's accuracy

OA: overall accuracy

\begin{tabular}{|c|c|c|c|c|}
\hline & & EMSR242 & EMSR230 & EMSR259 \\
\hline \multirow[t]{3}{*}{ PA } & Total area & 0.97 & 0.97 & 0.95 \\
\hline & Burned area & 0.96 & 0.96 & 0.93 \\
\hline & Unburned area & 0.97 & 0.97 & 0.97 \\
\hline \multirow[t]{3}{*}{ UA } & Total area & 0.96 & 0.96 & 0.95 \\
\hline & Burned area & 0.94 & 0.93 & 0.93 \\
\hline & Unburned area & 0.98 & 0.98 & 0.97 \\
\hline \multicolumn{2}{|c|}{ OA } & 0.97 & 0.97 & 0.96 \\
\hline \multicolumn{2}{|c|}{ Карра } & 0.93 & 0.93 & 0.90 \\
\hline
\end{tabular}

\section{Discussion and conclusion}

The approach presented here produced some initial reliable burned area maps using Sentinel2 imagery, by applying a standard band index. It is based on open programming libraries and open satellite imagery. What sets it apart from other approaches is the fully automated workflow, which is implemented in a cloud environment and triggered by a user, enabling fast and independent processing without human interaction. Moreover, the approach facilitates scalability, which could become important when the service is no longer triggered by a user but through event-detecting algorithm scanning, i.e. disaster alert systems. However, other studies have pioneered wildfire mapping by providing detailed descriptions of the representation of burned areas in the various bands (Huang, et al., 2016) and by developing burned area indices like the NBR and BAI (Key \& Benson, 2006). Existing open source tools facilitated the development of the algorithm. 
A comparison with the reference maps provided by the Copernicus EMS shows that the sets of results are similar. However, differences may occur due to the use of different imagery as well as a potentially different approach for burned area detection, which is not documented for the EMS. A detailed quality assessment would require access to VHR imagery for a visual comparison. The resampling to $500 \mathrm{~m}$ showed good results for the study areas. However, the algorithm needs to be tested and resampling adapted for smaller areas as well.

In I-REACT, it is planned to use the output of the algorithm for the calibration of wildfire forecasting and nowcasting during a wildfire event. These forecasts and nowcasts are needed to support fire fighters in attacking and extinguishing fires as quickly as possible. In the case of an enduring fire, the burned area maps will be one source of information that serves to update and improve the fire forecast. In addition, the maps can be used for validating the simulation models. An improvement of the service could be achieved by using Sentinel-3 imagery, which has a spatial resolution of $300 \mathrm{~m}$ but a daily revisit rate (ESA, 2018), thus increasing the availability of cloud-free scenes.

\section{Acknowledgement}

Our work is part of I-REACT, a European research project that has received funding from the European Union's Horizon 2020 research and innovation programme under grant agreement No. 700256.

\section{References}

Barredo, J. (2014). Expert's sub-Group 1: Identification of forest ecosystem services. Forest Europe: Expert Group and Workshop on a pan-European approach to valuation of forest ecosystem services.

Barredo, J., Bastrup-Birk, A., Teller, A., Onaindia, M., Fernàndez de Manuel, B., Madariaga, I., . . . Mimo, S. (2015). Mapping and assessment of forest ecosystems and their services - Applications and guidance for decision making in the framework of MAES. EUR $27751 \mathrm{EN}$.

Camia, A., Durrant, T., \& San-Miguel-Ayanz, J. (2013). Harmonized classification scheme of fire causes in the EU adopted for the European Fire Database of EFFIS. European Union.

Chavez, P. (1988). An Improved Dark-Object Substraction Technique for Atmospheric Scattering Correction of Multispectral Data. Remote Sensing of Environment, 24, pp. 459-479.

Chen, X., Vogelmann, J., Rollins, M., Ohlen, D., Key , C., Yang, L., . . . Shi, H. (2011). Detecting postfire burn severity and vegetation recovery using multitemporal remote sensing spectral indices and field-collected composite burn index data in a ponderosa pine forest. International Journal of Remote Sensing, 32(23), pp. 7905-7927.

Chuvieco, E., Martin, M., \& Palacios, A. (2002). Assessment of different spectral indices in the rednear-infrared spectral domain for burned land discrimination. International Journal of Remote Sensing, 23, pp. 5103-5110.

Congalton, R., \& Green, K. (2009). Assessing the Accuracy of Remotely Sensed Data: Principles and Practice. Boca Raton: CRC press.

EEA. (2002). Europe's biodiversity - biogeographical regions and seas. Retrieved 11.03.2018, from https://www.eea.europa.eu/publications/report_2002_0524_154909 
EEA. (2018). European Environment Agency. Retrieved 26.01.2018, from Biogeographical regions: https://www.eea.europa.eu/data-and-maps/data/biogeographical-regions-europe-3

ESA. (2018). Revisit and Coverage. Retrieved 28.01.2018, from Sentinel Online: https://sentinels.copernicus.eu/web/sentinel/user-guides/sentinel-2-msi/revisit-coverage

Huang, H., Roy, D., Boschetti, L., Zhang, H., Yan, L., Kumar, S. S., . . . Li, J. (2016). Separability Analysis of Sentinel-2A Multi-Spectral Instrument (MSI) Data for Burned Area Discrimination. Remote Sensing, 8.

IPCC. (2013). Climate Change 2013: The Physical Science Basis. Contribution of Working Group I to the Fifth Assessment Report of the Intergovernmental Panel on Climate Change. (T. Stocker, D. Qin, G.-K. Plattner, M. Tignor, S. Allen, J. Boschung, . . . P. Midgley, Eds.) Cambridge, United Kingdom and New York, NY, USA: Cambridge University Press.

Key, C., \& Benson, N. (2006). Landscape Assessment (LA). FIREMON: Fire effects monitoring and inventory system. . Fort Collins, CO: Gen. Tech. Rep. RMRS-GTR-164-CD. U.S. Department of Agriculture. Forest Service, Rocky Mountains Research Station.

Moreno, M. (2014). Forest Fires under Climate, Social and Economic Changes in Europe, the Mediterranean and Other Fire-Affected Areas Of The World (FUME Project). Retrieved 01 28, 2018,

from https://www.researchgate.net/profile/Minchao_Wu/publication/285601002_Threats_of_project ed_changes_in_fire_regime_for_newly_affected_areas_in_Europe_and_Northern_Africa/links/5 662215e08ae4931cd5c9969.pdf?inViewer=0\&pdfJsDownload=0\&origin=publication_detai

MunichRe. (2018). Munich Re. Retrieved 01.28.2018, from Press Release: https://www.munichre.com/en/media-relations/publications/press-releases/2018/2018-01-04press-release/index.html

Roy, D., Jin, Y., Lewis, P., \& Justice, C. (2005). Prototyping a global algorithm for systemtic fireaffected area mapping using MODIS time series data. Remote Sensing of Environment, 97, pp. 137-162.

Smith, A. M. S., Wooster, M. J., Powell, A. K., \& Usher, D. (2002). Texture based feature extraction: application to burn scar detection in Earth observation satellite sensor imagery. International Journal of Remote Sensing, 23, pp. 1733-1739.

Veraverbeke, S., Harris, S., \& Hook, S. (2011). Evaluating spectral indices for burned area discrimination using MODIS/ASTER (MASTER) airborne simulator data. Remote Sensing of Environment, 115, pp. 2702-2709. 